\title{
Markov Logic Based Inference Engine for CDSS
}

\author{
IMRAN SARWAR BAJWA*, BUSHRA RAMZAN*, AND SHABANA RAMZAN* \\ RECEIVED ON 26.08.2015 ACCEPTED ON14.12.2015
}

\begin{abstract}
CDSS (Clinical Decision Support System) is typically a diagnostic application and a modern technology that can be employed to provide standardized and quality medical facilities to the medical patients especially when expert doctors are not available at the medical centres. These days the use of the CDSSs is quite common in medical practice at remote areas. A CDSS can be very helpful not only in preventive health care but also in computerized diagnosis. However, a typical problem of CDSS based diagnosis is uncertainty. Typically, an ambiguity can occur when a patient is not able to explain the symptoms of his disease in a better way. The typically used forward chaining mechanisms in rule based decision support systems perform reasoning with uncertain data. ML (Markov Logic) is a new technique that has ability to deal with uncertainty of data by integrating FOL (First-Order-Logic) with probabilistic graphical models. In this paper, we have proposed the architecture of a ML based inference engine for a rule based CDSS and we have also presented an algorithm to use ML based forward chaining mechanism in the proposed inference engine. The results of the experiments show that the proposed inference engine would be intelligent enough to diagnose a patient's disease even from uncertain or incomplete/partial information.
\end{abstract}

Key Words: Clinical Decision Support System, First Order Logic, Markov Logic, Inference Engine.

\section{INTRODUCTION}

I $\mathrm{n}$ last couple of decades, the CDSS has been successfully used for diagnosis of diseases by physicians to improve the health care process [1] especially for patient of the remote areas. Typically, a CDSS can be knowledge-based or algorithm based. In this paper, we aim to use a knowledge-based CDSS for virtual telemedicine [1]. However, a key problem in using a CDSS for telemedicine is handling of uncertainty. Uncertainty exists practically in every phase of clinical decision making process [2]. Here, uncertainties and ambiguities in patient's data may occur due to patient's inability of describing accurate or complete information regarding what happened to the patient and how he felt; or doctors and care takers cannot tell exactly what they have observed about patient.

One of the main challenges here is how to realistically handle the possible uncertainties so that a CDSS can support clinical experts to make correct and reliable diagnosis and treatment decisions. The aim of this paper is to study mechanisms to perform reasoning with uncertain data. A few efforts [1-3] have been made to deal

* Department of Computer Science \& Information Technology, The Islamia University, Bahawalpur.

Mehran University Research Journal of Engineering \& Technology, Volume 36, No. 1, January, 2017 [p-ISSN: 0254-7821, e-ISSN: 2413-7219] 
with the problem of knowledge uncertainty in recent years, including stochastic logic programs [3], probabilistic relational models [4], Bayesian logic programs [5], relational dependency networks [6], and others. Such approaches typically combine probabilistic graphical models with a subset of FOL (e.g. Horn clauses), and can be quite complex. Recently, Richardson and Domingos [7] have introduced ML, a language that is conceptually simple, yet provides the full expressiveness of graphical models and FOL in finite domains. ML extends FOL by attaching weights to formulas However, most of the above mentioned approaches have some problems of applicability in real domains, mainly because its complexity and domain restrictiveness. Recently, a new area of research, called ML has emerged. ML has proven to provide interesting capabilities on learning and reasoning about uncertainty in many real world domains. ML is a novel representation formalism that combines FOL with probabilistic graphical models.

In this paper, we have proposed architecture of an inference engine that is based on ML for CDSS. In practice, our inference engine must cope with uncertainties in the data, uncertainties about which characteristics are most useful for handling the data, uncertainties in the associations between variables, and uncertainties in the value of the action that is used as a result of inference.

\section{RELATED WORK}

\subsection{Markov Logic}

Recent work on event pair wise comparison neglects logical constraints among temporal relations of different types that are believed to be supportive. Therefore, ML model is proposed that jointly asserts relations of all relation types concurrently [8]. The Evaluation of this model on the TempEval data shows that ML leads to about 2\% higher accuracy for all types of relations and demonstrate best results for the work while comparison to those of machine learning based systems. In this paper we presented a novel approach that uses ML to integrate both local features and global transition rules between temporal relations. The global ML model reaches the highest totals stated so far for the tasks. The temporal transition rules can also be realized by using an ILP (Integer Linear Programming) approach [9], ML holds many advantages. First of all, management of "rules of thumb" amid less specific temporal relations (such as OVERLAP or VAGUE) is Forthright. ML Engine itself learns weights for these rules. Another advantage is that reduced engineering overhead is to perform, as generation of ILPs for each document is not needed. To overcome the problem of meagerness and inconsistency of the data, methods for unsupervised learning in ML are used along with external or untagged data [10].

The author discourses the problem how it is possible that statistical learning algorithms can be incorporated into a broader AI (Artificial Intelligence) system both from a real-world engineering viewpoint and from the viewpoint of accurate depiction, learning, and reasoning [11]. Their intention is to build an assimilated intelligent system that can conglomerate pragmatic facts, hand-written rules, erudite rules, and erudite classifiers to accomplish combined learning and reasoning. They have applied a ML approach to integrate various learning components into the CALO system [12]. It assimilates several learning components with a ML inference engine, so that the components can get advantage from each other's prophecies. They have present two designs of the learning and reasoning layer in CALO: the MPE (Most Probable Explanation) Architecture and the Marginal Probability Architecture. They plan to develop connected, incremental algorithms constructed on the weight erudition and rule learning algorithms currently implemented in the ALMS (Alchemy Markov Logic System) [13].

Mehran University Research Journal of Engineering \& Technology, Volume 36, No. 1, January, 2017 [p-ISSN: 0254-7821, e-ISSN: 2413-7219] 


\subsection{Clinical Decision Support Systems}

DSSs are computer applications which depict certain types of expertise that can solve certain types of problems. Decision support systems are gaining an increased recognition in various domains, including business, engineering, military and medicine domain.

Variety of systems is available at the present days that can potentially sustenance clinical decisions. CDSSs are computer systems premeditated to influence clinician decision making about diseases of patients individually at the same point in time that these required decisions are made. The Fig. 1 shows a typical architecture of CDSS.

Most of presently available knowledge-based CDSS comes out of earlier expert systems research, where the objective was to develop a computer program that can act out on human intellect [14]. Medical science was one of top domains in which all these ideas can be applied [15]. In the last two decades, the designers of these systems have started to adapt them as they can be used more conveniently to support patient care practices in reality [16]. It has been discussed that most of the initial systems were diagnostic decision support systems. The finest accessible clinical knowledge is well organized, available to clinicians, and captured in an arrangement that expedites effective help for the whole decision making process.

\subsection{Inference Engine}

An inference mechanism or an inference engine is a software system that is premeditated for inducement of inference by exploring problems in the light of expert's knowledge which is provided in the knowledge base of any expert system. An inventive method for evaluation of the material's choice can be utilized as a possible part of an inference engine for an expert system in selection of material [17-20]. Since, experience plays an immense role in the material choice. The person who is experienced in the materials engineering field, it is observed that he will always prefer choosing the materials which he knows better and reject all other possibilities of choice, excluding and neglecting the new materials and loosing, in this manner, although the other choices might be more sound, both technically and economically [18]. In any expert system, the inference engine is the most important portion that performs the mechanism of "the thinking" by depending upon the contents of the knowledge base. It is proved that the method of materials choice can be viewed as one of an actual option for building an inference engine of an expert system in the materials selection [17].

\section{USED METHODOLOGY}

In order to apply the AI in a CDSS, a newly emerging technique has been used that is called as ML. The ML deals particularly with the vagueness and uncertainty. Uncertainty and ambiguity in natural clinical guidelines are very common complications which are difficult to

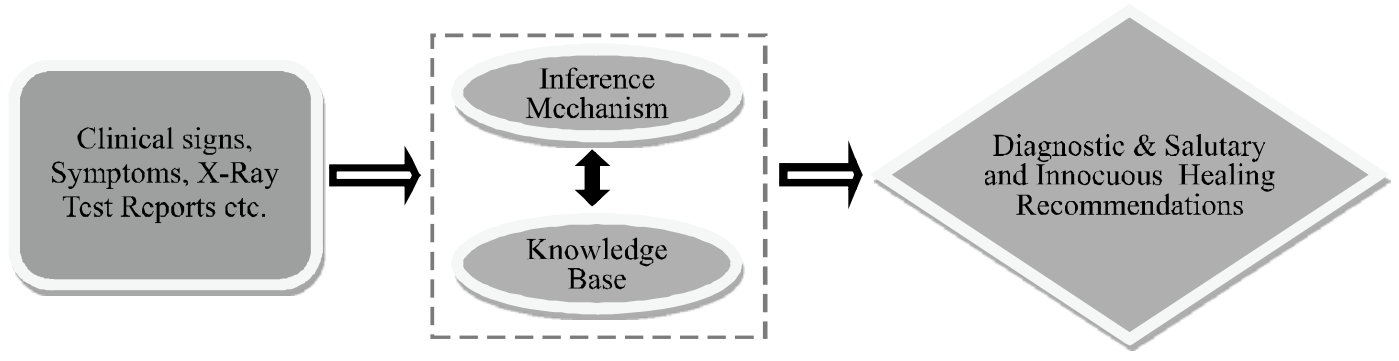

FIG. 1. A TYPICAL CDSS ARCHITECTURE

Mehran University Research Journal of Engineering \& Technology, Volume 36, No. 1, January, 2017 [p-ISSN: 0254-7821, e-ISSN: 2413-7219] 
handle. ML seems to be the only elucidation for the treatment of uncertainty in decision support systems. ML is used for describing the uncertainty and inaccuracy in particular mathematical language, unambiguously representing clinical uncertainty. The knowledge base contains the domain knowledge which is often, but not always, in the form of IF-THEN rules. The second part of the CDSS is called the inference engine which contains the formulae for combining the rules or relations in the knowledge base with actual input data that is the patient signs and symptoms. Finally, there has to be a communication mechanism between the operator/user and system, a way of inputting the patient data into the system and getting the output of the system to the user who will make the actual decision.

\subsection{Forward Chaining Inference Engine}

An inference engine is developed for a rule-based system whose basic components are Attributes, Conditions, Rules, and Actions. An Action is only needed to execute when a rule containing it is fired. Rule is only fired when all of its conditions are satisfied. The whole phenomenon involves assigning values to variables, evaluating conditions, and verifying if all of the conditions in a rule are satisfied. A general algorithm can be like below:

While values are input for the variables

Read value

Values are assigned to the variables

Check conditions

Fire rules whose conditions are satisfied

Example-1: If following are the rules;

Rule-1. IF X has temperature THEN X has fever.

Rule-2. IF Y has temperature AND Y has runny nose THEN Y has fever and flu.

The inference engine will preprocess the rules and extract the following conditions;:
Condition-1: $\quad \mathrm{X}$ has temperature

Condition-2: $\quad$ Y has temperature

Condition-3: $\quad$ Y has runny nose

The resulting Actions are

Action-1: $\quad \mathrm{X}$ has fever

Action-2: $\quad \mathrm{Y}$ has fever

Action-3: $\quad$ Y has flu

A data structure is presented in the form of a graph which shows the relationship between the attributes, conditions, rules, and actions in Fig. 2.

Knowledge-based CDSSs operate on IF-THEN rules, using compiled data and rules for making sense of that data.

\subsection{Rule Control}

In the literature of the Decision support systems, two problems in the control section were identified and the problems are lectured by the inference engine. However, there is a possibility that in a particular situation, there can be two or more rule available to run. Here, an inference engine has to decide in such situation to finalize a single rule to run.
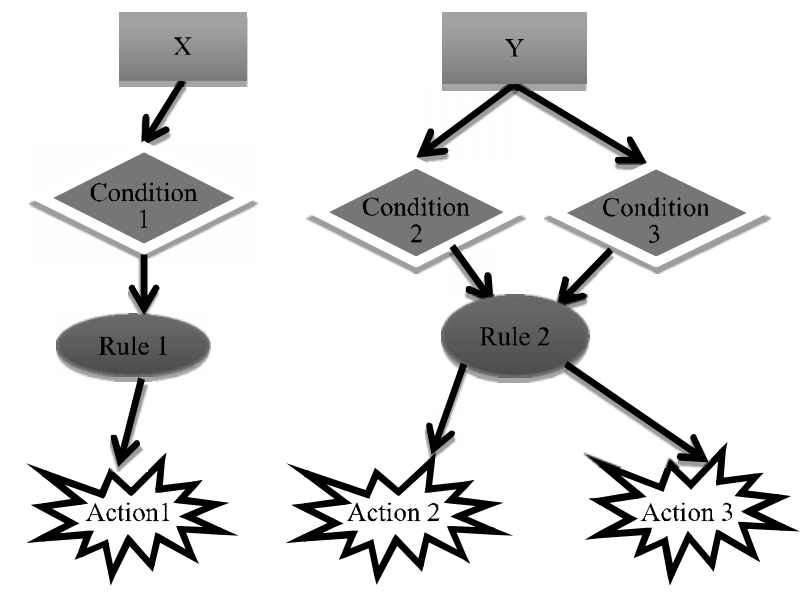

FIG. 2. USED DATA STRUCTURE FOR CDSS

Mehran University Research Journal of Engineering \& Technology, Volume 36, No. 1, January, 2017 [p-ISSN: 0254-7821, e-ISSN: 2413-7219] 


\subsection{Rule Selection Process}

An Inference engine is also called as a Rule Interpreter sine the task is to find and match rules to the requirements and then fine those particular rules. Following are typical steps followed in the rule selection process:

(i) First of all, one needs to match the available patterns of a rule against the factual data and finalize the rule.

(ii) If there are more than one rule available in a scenario the inference engine should select one of the conflicting rules and if none of the suitable rule is found, stop the processing.

(iii) Next step is the application of the selected rule. A rule can be applied by incorporating a new item to the working memory or deleting an existing item. Here the process is toped, if the termination condition is satisfied.

Forward chaining looks at the IF part of a rule first. Once all of the conditions are, met then the appropriate rule is chosen. A very simple forward chaining pseudo code would be as under

\section{Repeat}

(i) Find all rules whose conditions are satisfied with the given facts in working memory.

(ii) Choose one rule to fire, using any of the conflict resolution strategies.

(iii) Perform actions in conclusion; probably altering (add/remove) facts in working memory define by the consequent of the rule.

(iv) Flag the rule that has been fired
An algorithm is presented here which determine sequentially the working of the proposed inference engine of the clinical decision support system by defining different subroutines. The algorithm proceeds from a given situation to a desired goal, adding or removing new facts found.

\subsection{Forward Chaining Algorithm}

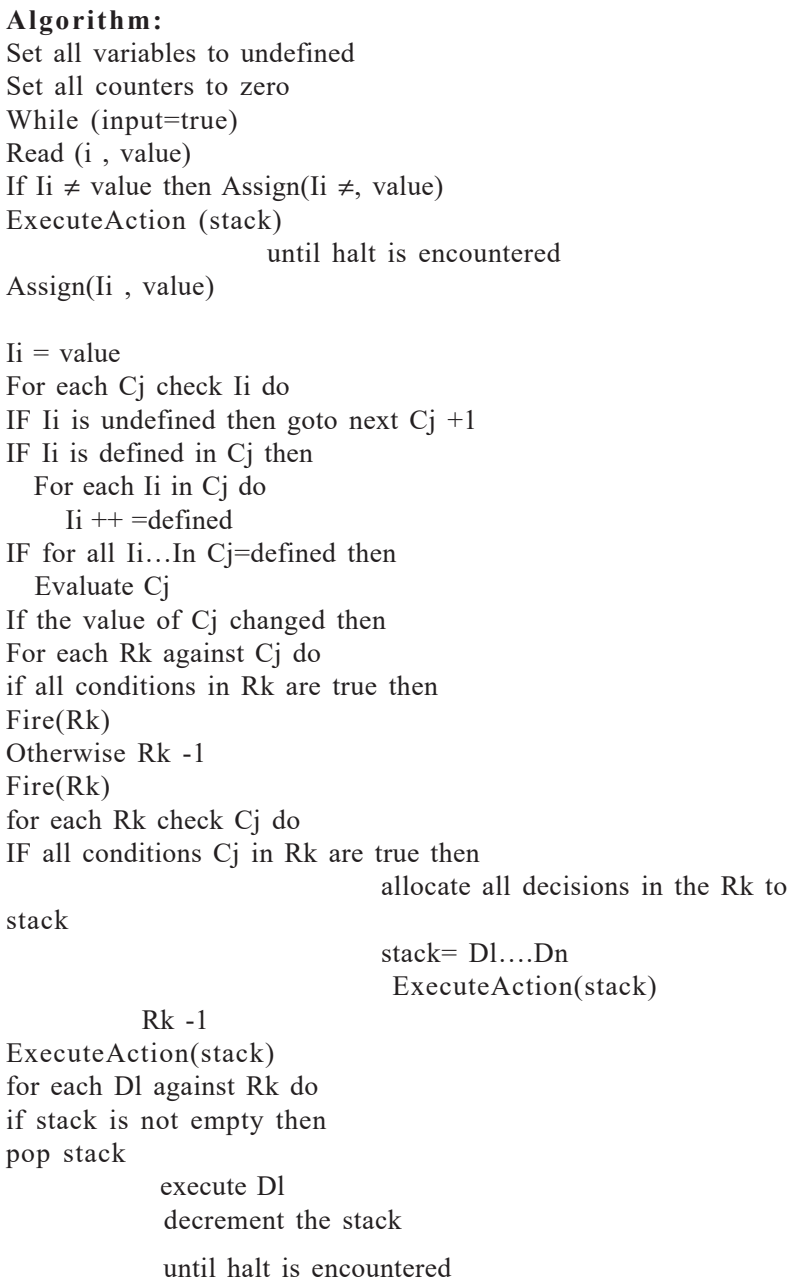

Firstly, the knowledge base is searched to find a rule that's IF part (condition) matches data in the database, then the rule is fired. That rule might have the desired solution. Rules must have the goal in their THEN (consequent or decision) parts. The working of the forward chaining algorithm can be explained by the following example

Mehran University Research Journal of Engineering \& Technology, Volume 36, No. 1, January, 2017 [p-ISSN: 0254-7821, e-ISSN: 2413-7219] 
Suppose the knowledge base contains the following Rule set (Fig. 3).

Rule (a): If I and $\mathrm{K}$ Then $\mathrm{N}$

Rule (b): If I and M Then O

Rule (c): If J Then M

Rule (d): If G Then L

Initial facts given by the User are: If I and $\mathrm{J}$ are true then is $\mathrm{L}$

\section{Solution}

Start with input given I, J is true and then

Inference Engine starts at Rule (a) and go forward/down the knowledge base till a rule "fires" is found.

\section{First Cycle}

Rule c fires: conclusion M is true.

New knowledge found.

No other rule fires.

End of first iteration.

Goal not found.

New knowledge found is that $\mathrm{M}$.

Go for second iteration.

Second Cycle:

Rule $\mathrm{b}$ fires: conclusion $\mathrm{O}$ is true.

New knowledge found.

Rule $\mathrm{d}$ fires: conclusion $\mathrm{L}$ is true.

$\mathrm{L}$ is true Goal found

Proved

\subsection{Markov Logic Based Inference Engine Architecture}

Generally, the major responsibility of the inference engine is to accomplish the rational process and the inference mechanism depending upon the information or rules contained in the knowledge base. There are some other significant components that are needed to run the overall system. Following is the way, Markov network represents the joint distribution:

$$
P(X=x)=\frac{1}{Z} \prod_{k} \phi_{k}\left(x_{\{k\}}\right)
$$

Here, the joint distribution (Pearl, 1988) of a model is represented as a set of variables i.e. $X_{\epsilon}\left(X_{1}, X_{2}, \ldots, X_{n}\right)$. In a typical network of $\mathrm{ML}$, a set of pair $\left(\mathrm{F}_{\mathrm{i}}, \mathrm{w}_{\mathrm{i}}\right)$ is used to represent a predicate and a predicate in first order logic is represented by $F_{i}$ and a real number depicts $\mathrm{w}_{\mathrm{i}}$ that is weight of the predicate/formula.

To update the weights of the used formula, statistical relational learning approach is incorporated by combining probability with the traditional FOL. Here, a typical MLN (Markov Logic Network) with a set of weights and formulas can be represented as below:

$$
P(X=x)=\frac{1}{Z} \operatorname{Exp}\left(\sum_{j} w_{i} f_{i}(x)\right)
$$

The weights of the formulas are dynamically updated by using diagnolized Newton Method (Pearl, 1988). Here, the weight update formula is:

$\mathrm{w}=\mathrm{w}+\mathrm{D}^{-1} \mathrm{~g}$

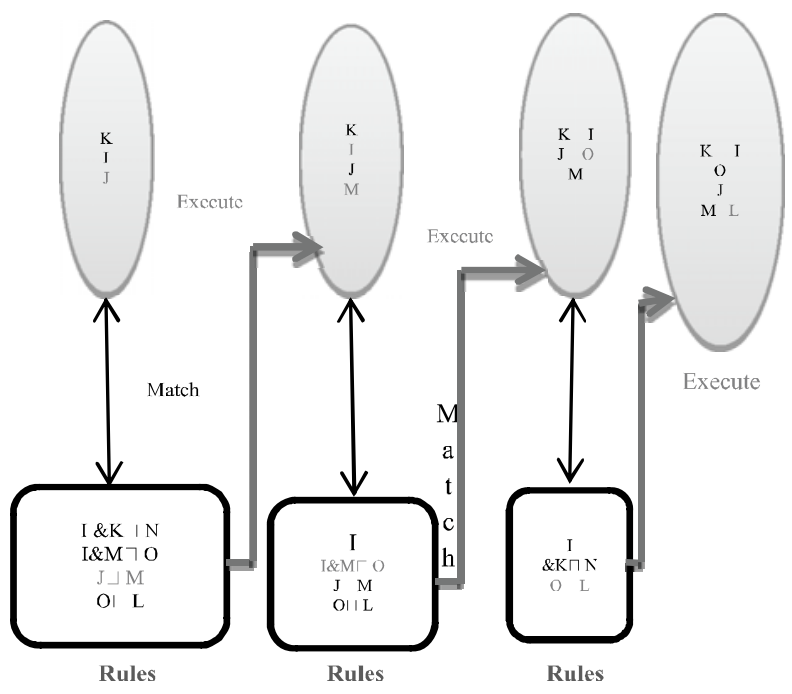

FIG. 3. FORWARD CHAINING PROCESS USED IN CDSS

Mehran University Research Journal of Engineering \& Technology, Volume 36, No. 1, January, 2017 [p-ISSN: 0254-7821, e-ISSN: 2413-7219] 
The other components of the architecture are as under

User

- $\quad$ User Interface

- $\quad$ Working Memory

- $\quad$ Knowledge Base

- Inference Engine

- $\quad$ Rule Loader

- $\quad$ Rule Organizer

- $\quad$ Markov Logic Reasoning Network

- $\quad$ Rule Matching

- $\quad$ Conflict Set

- $\quad$ Conflict Resolution

- $\quad$ Execute Action

\section{EXPERIMENTS AND RESULTS}

The used methodology is explained by the implementation of the ML based inference engine in the CDSS based Framework of telemedicine system proposed by [1]. Experiments are performed for the evaluation of the presented approach.

\subsection{Experiment Details}

To demonstrate the performance evaluation of the proposed ML based inference engine embedded in a CDSS, we have performed number of experiments using the developed system. We are going to present some of the real Sample case studies There are many phases from which the proposed system went through to solve a certain clinical case study.

- $\quad$ NL(Natural Language) Clinical Data Acquisition Phase

- NL Data Processing Phase

- Markov Logic Weight Assignment Phase

- Diagnosis Phase

\subsection{NL Clinical Data Acquisition Phase}

To perform the practical experiment, the first and foremost step is to obtain the clinical data about a certain patient's case study. A set of patient reports were collected from the BVH (Bahawalpur Victoria Hospital) outdoor section and selected reports were used to prepare patient's discretion in the English langauge. A sample example with the problem statement of the case study is given below.

Personal Details: The name of the patient is Ali. The gender of the patient is Male. The age of the patient is 43 years.

Vital Signs: The weight of the patient is $88 \mathrm{Kg}$. The patient's body temperature is $100 \dot{\mathrm{F}}$. The heart beat rate of the patient is $140 \mathrm{bpm}$. The blood pressure of patient is 145/110. The Respiration of the patient is 11 breaths per minute. The fasting sugar value of the patient is $120 \mathrm{mgdl}$.

The output obtain from this phase of the experiment is the patient report, which is in the NL format i.e. English.

\subsection{NL Data Processing Phase}

As the patient report acquired in the data acquisition phase is obtained in the NL format. We have used four steps to process a NL text file.

Lexical Analysis: The English written patient report obtained in the data acquisition phase is now available in the form of a paragraph which contains number of strings. These strings are tokenized into tokens or lexicons. These tokens are further gone through POS (Part-Of-Speech) tagger to get different parts of speech called as morphemes. Afterwards morphemes are lexically analyzed by a parse tree is shown in Table 1.

Syntax Analysis: In this phase of Analysis, all the sentences and the phrases of the paragraph of patient reports are authenticated in consultation with the defined

Mehran University Research Journal of Engineering \& Technology, Volume 36, No. 1, January, 2017 [p-ISSN: 0254-7821, e-ISSN: 2413-7219] 
grammatical rules in the English language. The parse tree generated is shown as under.

Input: The name of the patient is Ali.

\section{Parse Tree}

ROOT

(S

$$
\begin{aligned}
& \text { (NP } \\
& (\mathrm{NP} \text { (DT the) }(\mathrm{NN} \text { name })) \\
& (\mathrm{PP} \text { (IN of) } \\
& \quad(\mathrm{NP}(\mathrm{DT} \text { the })(\mathrm{NN} \text { patient }))) \\
& (\mathrm{VP}(\mathrm{VBZ} \text { is }) \\
& \quad(\mathrm{NP}(\mathrm{NN} \text { ali }))) \\
& (. .)))
\end{aligned}
$$

Semantic Analysis: By the semantic phase of analysis all the relations among various major parts of the sentences are documented in some sort of tabular form. Output of the Semantic analyzer for the above case study is given in Table 2.

\subsection{Symptoms Extraction}

The output of the semantic analyzer is used in this phase to extract the actual disease symptoms. It is shown in Table 3 .

\subsection{Markov Logic Weight Assignment}

ML has ability to handle uncertainty and learn from the data. The designed system, on the basis of these symptoms formulates the standard language rules with certain weights. The weights of the rules were randomly selected and these weights were adjusted to get the optimal output. In our experiments, we assigned a weight of 0.0 to 0.1 to the rules. Here, we can see Table 4 in which all the symptoms are shown with the weights assigned by the proposed CDSS system.

\subsection{Diagnosis Phase}

In this phase, the CDSS system detects and diagnoses the disease. The proposed CDSS system identifies the disease according to the assigned weights. On the basis of the CDSS diagnosis about the patient's disease, the system prescribes medication (including dose details) with diet details.

\section{RESULTS AND DISCUSSION}

To find out the accuracy the used framework of three gatherings of twenty patients were characterized. For every gathering three reports i.e. simple, normal and troublesome were created for every gathering. The side effect reports were painstakingly arranged and prepared for every patient utilizing the composed social insurance framework. For right and wrong determination of an indication report different focuses were given. Along the patient reports (data) received from $\mathrm{BVH}$, the diagnosis

\begin{tabular}{|c|c|}
\hline String & $\begin{array}{l}\text { The name of the patient is Ali. The gender of the patient is Male. The age of the patient is } 43 \text { years. The weight of the patient is } \\
88 \mathrm{Kg} \text {. The patient's body temperature is } 100 \dot{\mathrm{F}} \text {. The Pulse rate of the patient is } 140 \mathrm{bpm} \text {. The blood pressure of patient is } \\
145 / 110 \text {. The Respiration of the patient is } 11 \mathrm{bpm} \text {. The fasting sugar value of the patient is } 120 \text { mgdl. }\end{array}$ \\
\hline POS Tagging & $\begin{array}{l}\text { The/DT name/NN of/IN the/DT patient/NN is/VBZ Ali/NN ./. The/DT gender/NN of/IN the/DT patient/NN is/VBZ male/NN ./. } \\
\text { The/DT age/NN of/IN the/DT patient/NN is/VBZ 43/CD years/NNS ./. The/DT weight/NN of/IN the/DT patient/NN is/VBZ } \\
88 / \mathrm{CD} \mathrm{kg} / \mathrm{NNP} . / . \text { The/DT patient/NN 's/POS body/NN temperature/NN is/VBZ 100/CD ?/NNP ./. The/DT Pulse/NN rate/NN } \\
\text { of/IN the/DT patient/NN is/VBZ 140/CD bpm/NNP ./. The/DT blood/NN pressure/NN of/IN the/DT patient/NN is/VBZ } 145 / C D \\
\text { /// 110/CD ./. The/DT Respiration/NN of/IN the/DT patient/NN is/VBZ 11/CD bpm/NNP ./. The/DT fasting/NN sugar/NN of/IN } \\
\text { the/DT patient/NN is/VBZ 120/CD mgd/NNP ./. }\end{array}$ \\
\hline
\end{tabular}
of the experts were also collected. Here, the diseases identified by the deigned system were matched with the diagnosis results of the human medical experts and the results were classified into correct, incorrect and missing categories. Fig. 4 highlights the results.

TABLE 1. PART-OF-SPEECH TAGGING OF THE INPUT PATIENT REPORT

Mehran University Research Journal of Engineering \& Technology, Volume 36, No. 1, January, 2017 [p-ISSN: 0254-7821, e-ISSN: 2413-7219] 
ML attaches a numeric weight to each inference rules which are present in the knowledge base. The designed system very effective and have high accuracy up to $90.35 \%$. One limitation of our approach is that the weights of the formulas are manually assigned and hard to tune. Following are some benefits of using the proposed framework of CDSS:

TABLE 2. OUTPUT OF THE SEMANTIC ANALYZER

\begin{tabular}{|c|c|c|c|c|c|c|c|}
\hline No. & Chunks of Sentence & Syntax & Quantity & Logical & Type & Prep & EOS \\
\hline 1. & name patient & Subject & & & State & & \\
\hline 2. & Is & H.Verb & & & & & True \\
\hline 3. & Ali & Subject & & & Active & & \\
\hline 4. & gender patient & Subject & & & State & & \\
\hline 5. & Is & H.Verb & & & & & True \\
\hline 6. & Male & Subject & & & Active & & \\
\hline 7. & age patient & Subject & & & State & & \\
\hline 8. & Is & H.Verb & & & & & \\
\hline 9. & Years & Object & 43 & & & & True \\
\hline 10. & weight patient & Subject & & & State & & \\
\hline 11. & Is & H.Verb & & & & & \\
\hline 12. & $\mathrm{Kg}$ & Object & 88 & & & & True \\
\hline 13. & patient's body temperature & Subject & & & State & & \\
\hline 14. & Is & H.Verb & & & & & \\
\hline 15. & $\dot{F}$ & Object & 100 & & & & True \\
\hline 16. & pulse rate patient & Subject & & & State & & \\
\hline 17. & Is & H.Verb & & & & & \\
\hline 18. & Bpm & Object & 140 & & & & True \\
\hline 19. & blood pressure patient & Subject & & & State & & \\
\hline 20. & Is & H.Verb & & & & & True \\
\hline 21. & Patient & Subject & $145 / 110$ & & Active & & \\
\hline 22. & respiration patient & Subject & & & State & & \\
\hline 23. & Is & H.Verb & & & & & \\
\hline 24. & BPM & Object & 11 & & & & True \\
\hline 25. & fasting sugar value patient & Subject & & & State & & \\
\hline 26. & Is & H.Verb & & & & & \\
\hline 27. & Mgdl & Object & 120 & & & & True \\
\hline
\end{tabular}

Mehran University Research Journal of Engineering \& Technology, Volume 36, No. 1, January, 2017 [p-ISSN: 0254-7821, e-ISSN: 2413-7219] 
TABLE 3. LIST OF SYMPTOMS EXTRACTED

\begin{tabular}{|c|c|c|}
\hline No. & Symptoms & Value \\
\hline 1. & Name & Ali \\
\hline 2. & Gender & Male \\
\hline 3. & Age & 43 years \\
\hline 4. & Weight & $88 \mathrm{~kg}$ \\
\hline 5. & Body temperature & $100 ?$ \\
\hline 6. & Pulse rate & $140 \mathrm{bpm}$ \\
\hline 7. & Blood Pressure & $145 / 110$ \\
\hline 8. & Respiration & $11 \mathrm{BPM}$ \\
\hline 9. & Fasting sugar value & $120 \mathrm{mgdl}$ \\
\hline
\end{tabular}

TABLE 4. WEIGHTED LIST OF SYMPTOMS

\begin{tabular}{|c|c|c|c|}
\hline No. & Symptoms & Value & Weight \\
\hline 1. & Name & Ali & 1.0 \\
\hline 2. & Gender & Male & 1.0 \\
\hline 3. & Age & 43 years & 1.0 \\
\hline 4. & Weight & $88 \mathrm{~kg}$ & 0.8 \\
\hline 5. & Body temperature & $100 ?$ & 0.2 \\
\hline 6. & Pulse rate & $140 \mathrm{bpm}$ & 0.4 \\
\hline 7. & Blood Pressure & $145 / 110$ & 1.0 \\
\hline 8. & Respiration & $11 \mathrm{BPM}$ & 0.4 \\
\hline 9. & Fasting sugar value & $120 \mathrm{mgdl}$ & 0.2 \\
\hline
\end{tabular}

- Such system can helping improving the admittance of specialty care

- $\quad$ Reduced health care cost

- The emergency medical services can be upgraded

- $\quad$ There can be least reliance on the medical expert

- $\quad$ Automated patient record keeping

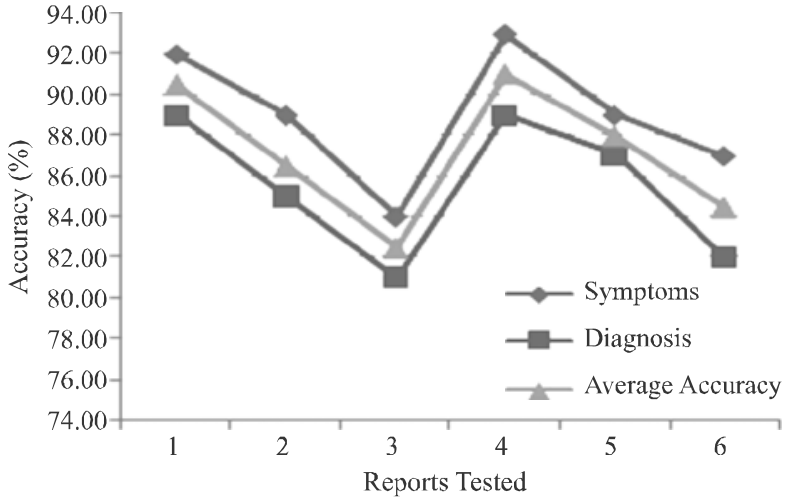

FIG. 4. RESULTS OF THE USED CDSS BASED ON MARKOV LOGIC

- Simple consultation based on automated diagnosis

- Entertaining extra queries with available resources.

\section{CONCLUSION}

CDSSs experts are concerned in diagnosis of diseases for their patients. But face some problems due to uncertainty in the patients' reports. Reasoning which is performed by inference engine. In this paper we have proposed ML based reasoning framework for the CDSS that can be successfully applied to solve a number of difficult and important patient reports containing uncertain and vague information.

\section{FUTURE WORK}

Humans are good at writing rules but not so good at assigning weights to the rules they write. In future work, we also are studying methods for allowing inference engine to learn its own rules and learn the weights automatically.

\section{ACKNOWLEDGEMENT}

Authors are thankful to the Bahawalpur Victoria Hospital, Bahawalpur, Pakistan, for providing us the support required in this study.

Mehran University Research Journal of Engineering \& Technology, Volume 36, No. 1, January, 2017 [p-ISSN: 0254-7821, e-ISSN: 2413-7219] 


\section{REFERENCES}

[1] Kareem, S., and Bajwa, I.S., "A Virtual Telehealth Framework", IEEE 7th International Conference on Applications in Emerging Technologies, pp. 1-6, September, 2011.

[2] Kong, G., Xu, D.L., and Yang, J.B., "Clinical Decision Support Systems", International Journal of Computational Intelligence Systems, Volume 1, No. 2, pp. $159-167,2008$.

[3] Johnson, M.P., Zheng, K., and Padman, R., "Modeling the Longitudinality of User Acceptance of Technology with an Evidence-adaptive Clinical Decision Support System”, Decision Support Systems, Volume 57, No. 1, pp. 444-453, January, 2014.

[4] Lu, J., Wu, D., Mao, M., Wang, W., and Zhang, G. "Recommender System Application Developments: A Survey”, Decision Support Systems, Volume 74, No. 1, pp. 12-32, June, 2015.

[5] Kersting, K., and De Raedt, L., “Towards Combining Inductive Logic Programming with Bayesian Networks", Inductive Logic Programming, pp. 118-131, 2001.

[6] Chambers, N., and Jurafsky, D., "Jointly Combining Implicit Constraints Improves Temporal Ordering", Conference on Empirical Methods in Natural Language Processing, pp. 698-706, October, 2008.

[7] Poon, H., and Domingos, P., "Joint Unsupervised Co-Reference Resolution with Markov Logic", Conference on Empirical Methods in Natural Language Processing, pp. 650-659, October, 2008.

[8] Musen, M.A., Middleton, B., and Greenes, R.A., "Clinical Decision-Support Systems", Biomedical Informatics, pp. 643-674, Springer London, 2014.
Addington, J., Shah, H., Liu, L., and Addington, D., "Reliability and Validity of the Calgary Depression Scale for Schizophrenia (CDSS) in Youth at Clinical High Risk for Psychosis", Schizophrenia Research, Volume 153, No. 1, pp. 64-67, 2014.

[10] Dietterich, T.G., and Bao, X., "Integrating Multiple Learning Components through Markov Logic", AAAI, pp. 622-627, July, 2008.

[11] Kok, S., and Domingos, P., "Statistical Predicate Invention", 24 ${ }^{\text {th }}$ International Conference on Machine Learning, pp. 433-440, June, 2007.

[12] Lowd, D., and Domingos, P., "Efficient Weight Learning for Markov Logic Networks", Knowledge Discovery in Databases, pp. 200-211, 2007.

[13] Badarau, G., Badarau, V., Ionita, I., and Stefan, M., "Diagnosis Method and Evaluation of the Metalic Materials Choice", Buletinul IPI Fasc. 1, Tom LI(LV), pp. 83-88, 2005.

[14] Martínez-Pérez, B., dela Torre-Díez, I., LópezCoronado, M., Sainz-de-Abajo, B., Robles, M., and García-Gómez, J.M., “Mobile Clinical Decision Support Systems and Applications: A Literature and Commercial Review", Journal of Medical Systems, Volume 38, No. 1, pp. 1-10, 2014.

[15] Gupta, A., Raja, A.S., and Khorasani, R. "Examining Clinical Decision Support Integrity: is Clinician SelfReported Data Entry Accurate?", Journal of the American Medical Informatics Association, Volume 2, No. 1, pp. 23-26, 2014.

[16] Imler, T.D., Morea, J., and Imperiale, T.F., "Clinical Decision Support with Natural Language Processing Facilitates Determination of Colonoscopy Surveillance Intervals", Clinical Gastroenterology and Hepatology, Volume 12, No. 7, pp. 1130-1136, 2014. 
[17] Brunette, M.F., Ferron, J.C., McHugo, G.J., Davis, K.E., Devitt, T.S., Wilkness, S.M., and Drake, R.E., "An Electronic Decision Support System to Motivate People with Severe Mental Illnesses to Quit Smoking”, Psychiatric Services, 2014.

[18] Bountris, P., Kotronoulas, G., Tagaris, T., Haritou, M., Spathis, A., Karakitsos, P., and Koutsouris, D., "CxCaDSS: A Web-Based Clinical Decision Support System for Cervical Cancer", 6th European International Conference on International Federation for Medical and Biological Engineering, pp. 757-760, January, 2015.
[19] Yin, Z., Min, L., Lu, X., and Duan, H., “A Clinical Decision Support System for Primary Headache Disorder Based on Hybrid Intelligent Reasoning”, Biomedical Engineering and Informatics, pp. 683-687, October, 2014.

[20] Marcum, J.A. “An Integrated Model of Clinical Reasoning: Dual Process Theory of Cognition and Metacognition", Journal of Evaluation in Clinical Practice, Volume 18, No. 5, pp. 954-961, 2012. 